\title{
A Clinical Evaluation of Definitive and Clinical Allergic Bronchopulmonary Mycosis
}

\author{
Hiroto Matsuse ${ }^{1}$, Hiroko Nakata ${ }^{1}$, Susumu Fukahori ${ }^{1}$, Tomoko Tsuchida ${ }^{2}$, Tetsuya Kawano ${ }^{3}$, \\ Shinya Tomari ${ }^{4}$, Chizu Fukushima ${ }^{5}$, Nobuko Matsuo ${ }^{6}$, Sadahiro Asai ${ }^{4}$ and Shigeru Kohno ${ }^{1}$
}

\begin{abstract}
Objective The present study aims to overcome problems associated with the early diagnosis of allergic bronchopulmonary mycosis (ABPM) using the current criteria.

Patients and Methods Clinical features including radiographic findings from 10 patients with definitive ABPM based on the diagnostic criteria of Rosenberg-Patterson were compared with those from 9 patients with ABPM clinically diagnosed by respiratory allergy specialists.

Results ABPM should be considered in patients with peripheral blood eosinophilia and pulmonary infiltration and/or central bronchiectasis when serum total IgE is elevated. Complication by bronchial asthma suggested ABPM, but was not essential. The expectoration of sputum containing solid components was a critical factor in patients with a history in ABPM. Evaluation of sputum cultures, serum specific IgE antibodies, skin tests and precipitating antibodies were required to establish a diagnosis, but the positive rate of these tests remained low.

Conclusions Even when a definitive diagnosis cannot be established, systemic corticosteroid therapy should be initiated for clinically diagnosed ABPM to prevent irreversible pulmonary dysfunction.
\end{abstract}

Key words: allergic bronchopulmonary mycosis, diagnostic criteria of Rosenberg and Patterson, pulmonary infiltration, peripheral blood eosinophlia

(DOI: 10.2169/internalmedicine.45.1751)

\section{Introduction}

Aspergillus organisms are typical and ubiquitous environmental fungi that can cause several forms of pulmonary disease in humans. Clinical features differ depending on whether the condition is saprophytic infestation, invasive or allergic diseases including Aspergillus-induced asthma, hypersensitivity pneumonia and allergic bronchopulmonary aspergillosis (ABPA). The latter was identified by Hinson et al in asthmatics with recurrent pulmonary infiltration, peripheral blood eosinophilia and whose sputum contained Aspergillus (1). The causative organisms are mainly fungal, especially Aspergillus fumigatus (Af), but can include others such as A. flavus, A. niger, Candida albicans, Penicillium and also Schizophylum commune (2-6). Thus, ABPA has recently been referred to as allergic bronchopulmonary mycosis (ABPM). Generally, ABPA is a reversible disease that responds well to systemic corticosteroid therapy. However, a subset of patients develops irreversible pulmonary dysfunction due to pulmonary fibrosis, especially when the initiation of corticosteroids is delayed, suggesting the importance of early diagnosis (7). Among several diagnostic criteria for ABPM (8-10), we included definitive ABPM based on the Rosenberg-Patterson criteria that are the most frequently applied in Japan. Patients with clinical ABPM that could not be diagnosed as definitive by the Rosenberg-Patterson criteria but who were clinically diagnosed as having ABPM by

\footnotetext{
${ }^{1}$ Second Department of Internal Medicine, Nagasaki University School of Medicine, Nagasaki, ${ }^{2}$ Department of Internal Medicine, Goto Central Hospital, Goto, ${ }^{3}$ Department of Internal Medicine, Senju Hospital, Sasebo, ${ }^{4}$ Department of Internal Medicine, Sasebo City General Hospital, Sasebo, ${ }^{5}$ Department of Internal Medicine, Nagasaki Kita Hospital, Nagasaki and ${ }^{6}$ Department of Internal Medicine, Nagasaki Municipal Medical Center, Nagasaki

Received for publication February 3, 2006; Accepted for publication March 22, 2006

Correspondence to Hiroto Matsuse, Second Department of Internal Medicine, Nagasaki University School of Medicine, 1-7-1 Sakamoto, Nagasaki 852-8501
} 
Table 1. Diagnostic Criteria of ABPA

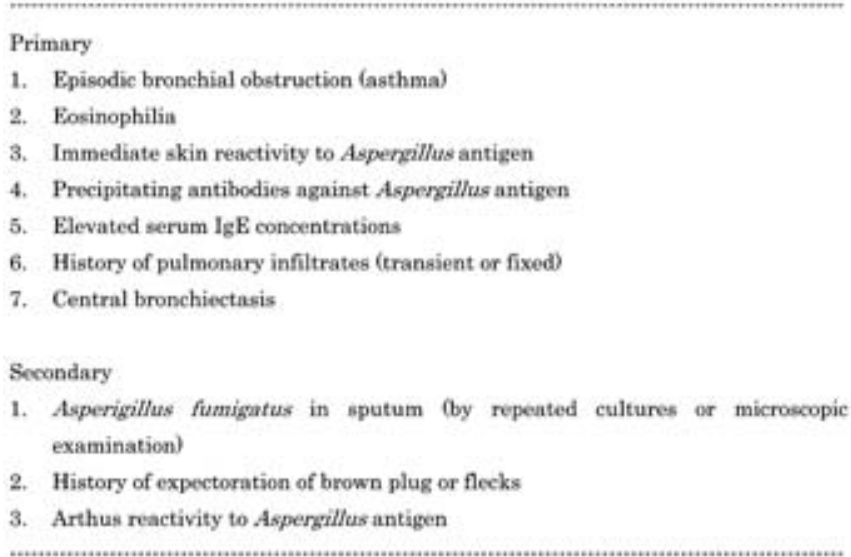

an attending specialist were also included and their clinical features were retrospectively compared to identified issues associated with an early diagnosis of ABPA using the current diagnostic criteria.

\section{Material and Methods}

\section{Patients}

Nineteen patients diagnosed with ABPM in 6 institutions in Nagasaki prefecture were classified as having definitive or clinical ABPM according to the following criteria. Definitive ABPM was assumed when 6 or more of the primary Rosenberg-Patterson criteria were positive (8) (Table 1). Positive findings for secondary criteria further confirmed the diagnosis, but were not essential. Patients with ABPM caused by fungi other than $A f$ were also included and $A f$ was substituted in the diagnostic criteria as the causative fungus. Clinical ABPM was diagnosed by a respiratory and/ or allergy specialist at each institute when definitive ABPM could not be established by the Rosenberg-Patterson criteria. Clinical ABPM was finally reevaluated by specialists in other institutions. A clinical diagnosis was determined when involvement of the immune system in response to the causative fungi was confirmed by sputum cultures, skin and serum tests in addition to pulmonary infiltration and/or central bronchiectasis. Other diseases were carefully excluded. Clinical features including radiographic findings of these patients were retrospectively evaluated from medical records before the initiation of systemic corticosteroid therapy. A subset of patients who had already been administered with oral corticosteroids to treat severe asthma was evaluated while under treatment with these drugs. The ethics committee of each institution approved the present study and all patients provided written informed consent to participate in all procedures associated with the study.

\section{Statistical analysis}

Results are expressed as means \pm standard deviation (SD). Positive ratios and mean values between clinical and defini-
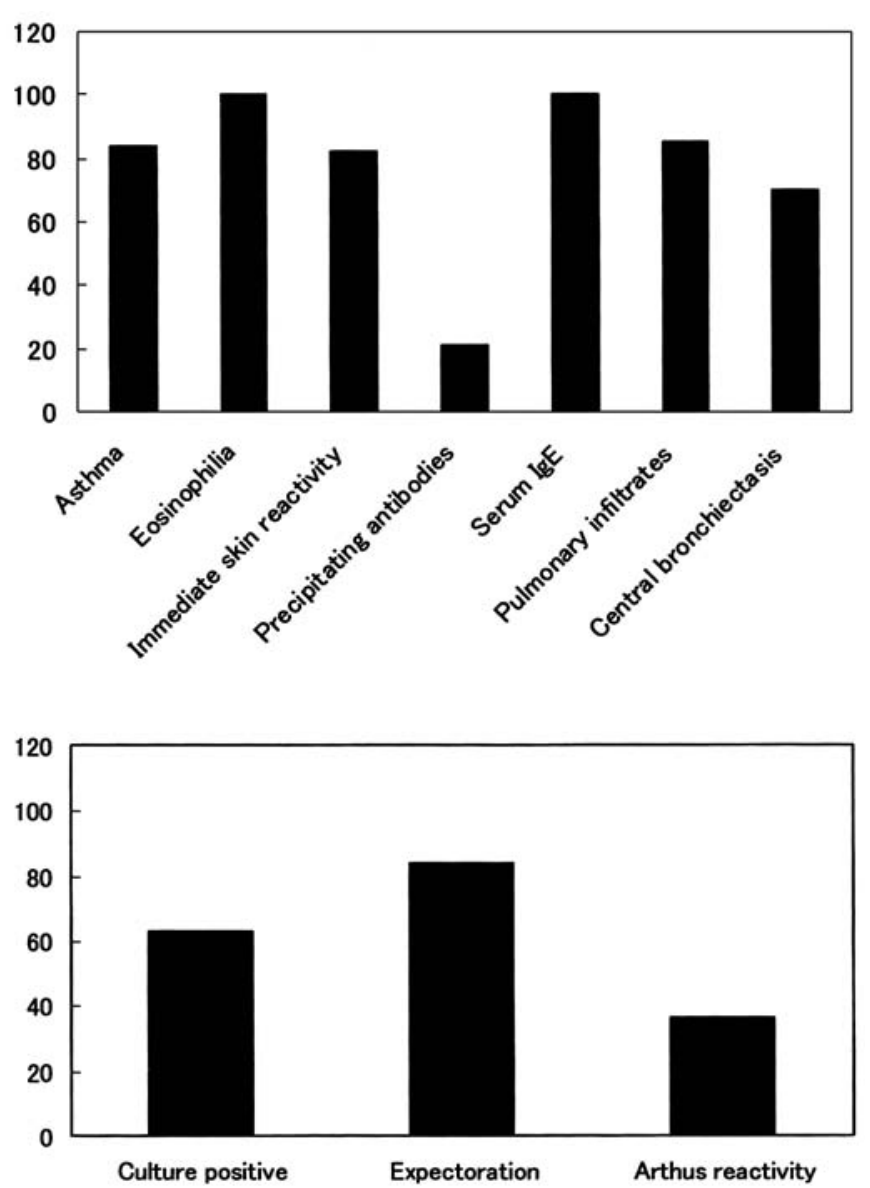

Figure 1. Positive rates of diagnostic criteria for ABPA (all patients, $n=19$ ). Positive rates of primary (upper) and secondary (lower) criteria.

tive ABPM were compared by the chi-square and Student's $\mathrm{t}$ tests, respectively. A value of $\mathrm{p}<0.05$ was considered significant.

\section{Results}

\section{Clinical features}

The mean age of the 12 male and 7 female patients was $48.3 \pm 16.6$ y (range 23 to $80 \mathrm{y}$ ). Within these, 10 were classified as definitive and the remaining 9 were considered to have clinical ABPM. Fig. 1 shows the clinical features of both definitive and clinical ABPM according to the Rosenberg-Patterson criteria. All of the patients had peripheral blood eosinophilia and increased serum total IgE levels (mean 5,007.0 $\pm 9,710.3 \mathrm{IU} / \mathrm{ml}$ ), suggesting that these criteria are essential for diagnosing ABPM. Three of the patients did not have asthma and the severity of complicating asthma in the other 9 patients varied. In addition to asthma, one patient had allergic rhinitis and another had eosinophilic otitis media. One patient had aspergilloma in addition to ABPM.

The positive rate of almost all primary criteria was $>80 \%$ except for central bronchiectasis $(70 \%)$ and precipitating antibodies $(21 \%)$. The positive rates of precipitating antibody 
Table 2. Patients' Characteristics

\begin{tabular}{lll} 
& Definitive & Clinical \\
\hline N & 10 & 9 \\
Age & $45.9 \pm 17.6$ & $51.0 \pm 16.0$ \\
Gender (M/F) & $6 / 4$ & $6 / 3$ \\
Eosinophils (/mm1)* & $1018.6 \pm 591.1$ & $1841.2 \pm 462.7$ \\
Total IgE (IU/ml) & $7381.6 \pm 13059.5$ & $2368.6 \pm 2324.7$ \\
Stage (EIIIIIIIV:V)* & $0: 1: 0: 5: 3: 1$ & $5: 300: 0: 1: 0$
\end{tabular}

* $P<0,05$

were lower than those of the secondary criteria and $84 \%$ of the patients expectorated mucous plugs (a secondary criterion), which was more frequent than the positive ratio of precipitating antibody. Skin was tested and/or serum $\operatorname{IgE}$ antibodies against $A f$ were determined in 17 patients. The immediate skin reaction and the Arthus reaction were positive in $48.5 \%$ and in $20.4 \%$ of the patients, respectively. Serum Af-specific antibody was positive in $40.7 \%$ and both the skin test and serum IgE antibody were positive in $36.7 \%$ of the patients. Neither test was positive in $40.8 \%$. Although 2 patients were negative for serum IgE antibodies, their skin tests were positive and all patients who were serum IgE positive were also positive for the skin test.

\section{Comparison of definitive and clinical ABPM}

Table 2 compares the characteristics of patients with definitive and with clinical ABPM. Age and gender did not significantly differ and total serum IgE levels were slightly, but not significantly higher in patients with a definitive diagnosis due to a wide variance. Peripheral blood eosinophil counts were significantly higher in patients whose diagnosis was clinical, rather than definitive. This was probably due to systemic corticosteroid administration for some definitive ABPM with severe corticosteroid-dependent asthma. An evaluation of clinical staging revealed that clinical ABPM was classified at a significantly earlier stage than definitive ABPM. The present study did not identify a significant difference in the positive rate of the Rosenberg-Patterson criteria between definitive and clinical ABPM, probably due to the small sample size. However, the immediate skin reaction against $A f$ and central bronchiectasis are probably more frequent in definitive ABPM (Fig. 2). These results suggest that clinical ABPM might include ABPM caused by fungi other than $A f$ seropositive, earlier ABPM that is not accompanied by central bronchiectasis.

\section{Discussion}

Allergic bronchopulmonary mycosis is an allergic pulmonary disease caused by environmental fungi such as $A f$. The positive rate of sputum culture for fungi is generally low and causative fungi can be obscure even when a sputum culture is positive. Fungi were cultivated from sputum from 14 (70\%) patients, but we could identify the causative fungi in
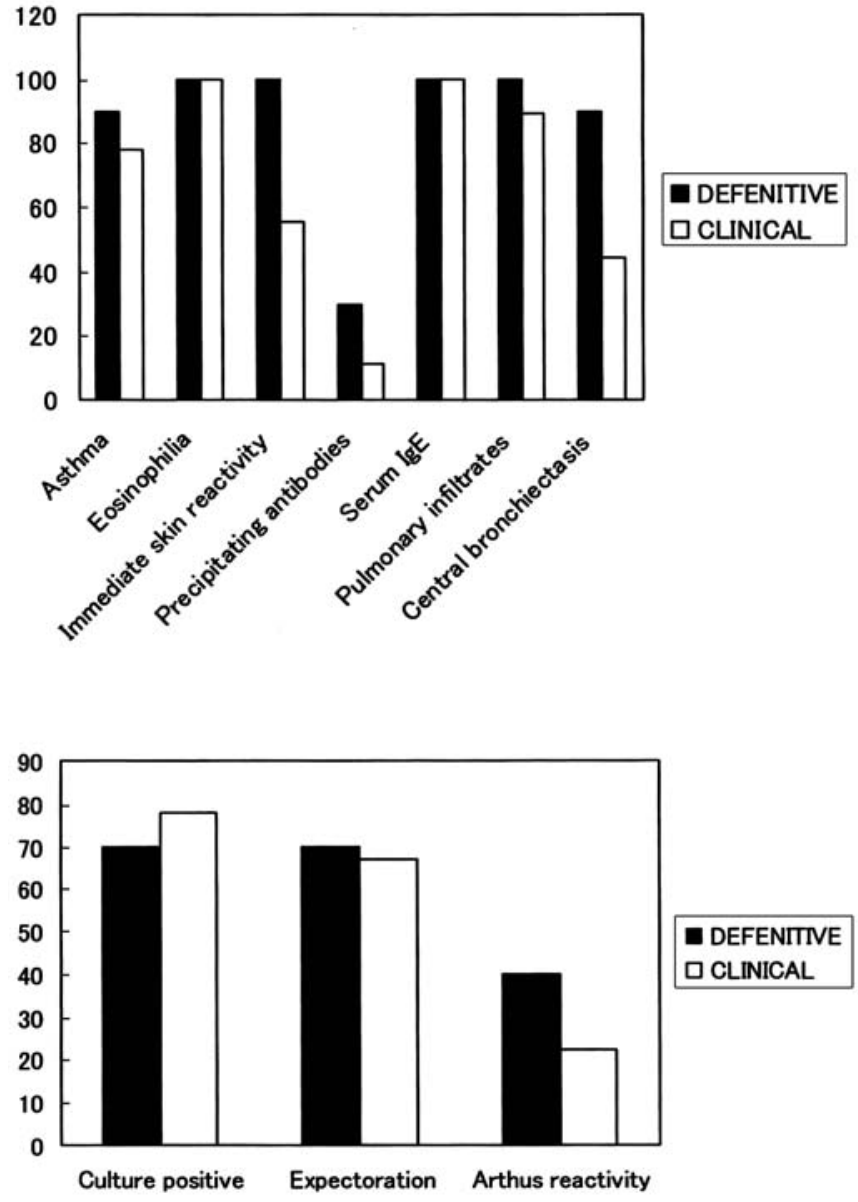

Figure 2. Comparison of definitive $(n=10)$ and clinical (n=9) ABPM. Positive rates of primary criteria (upper) and secondary (lower) criteria.

only $9(45 \%)$ of them. Even when the causative fungi are unknown, further immunological examination should be performed, which leads to difficulties in fulfilling all of the Rosenberg-Patterson diagnostic criteria. On the contrary, early diagnosis and the initiation of systemic corticosteroids are essential for the treatment of ABPM to prevent pulmonary fibrosis. Taken together, conventional and highly disease-specific clinical diagnostic criteria seem to be crucial.

The positive factors among the primary RosenbergPatterson criteria in the present study including both clinical and definitive ABPM were asthma (84\%), peripheral blood eosinophilia (100\%), immediate skin reaction to $A f$ antigen (82\%), precipitating antibody against $A f$ antigen (21\%), increased serum total $\operatorname{IgE}(100 \%)$, pulmonary infiltration $(85 \%)$ and central bronchiectasis $(70 \%)$. The factor with the lowest positive rate was serum precipitating antibody, whereas peripheral blood eosinophilia and increased serum total IgE were evident in all patients. Three (15\%) of the patients did not have asthma, a classically essential component of ABPA, suggesting that the presence of asthma is important but not essential to an early diagnosis of ABPM as previously thought $(11,12)$. The immediate skin test to $A f$ antigen was positive in a high ratio of patients. A serum 
antigen-specific antibody has frequently been substituted in recent skin tests. However, the results of the present study indicated that the skin test is more sensitive than the serum antibody level and thus should be performed when ABPM is suspected. A key finding among the secondary criteria was the high incidence of sputum expectoration. One review (13) identified sputum expectoration in $<20 \%$ of patients. Based on our clinical experience, a detailed interview of patients with ABPM could reveal a history of sputum expectoration containing solid components. In addition, fiberoptic bronchoscopy might be useful for finding mucous plugs within visible areas even when a patient does not have a history of sputum expectoration as reported (14).

Our comparison found that clinical ABPM had identifiably earlier stages (i.e., ABPM-S and stage I) than definitive APBM. Greenberger defined the clinical stages I, II, III, IV and $\mathrm{V}$ of ABPM as acute, remission, exacerbation, steroiddependent asthma and fibrosis, respectively (15). Furthermore, ABPA can be also classified as either ABPA-CB (central bronchiectasis) or ABPA-S (seropositive) based on the presence or absence of central bronchiectasis, a marker of tissue destruction. The present patients with clinical ABPM included many with ABPA-S and stage I, whereas those with definitive ABPM included many with recurrent pneumonia and with steroid-dependent severe asthma, who were thus defined as having stages III and IV. We suggest that clinical ABPM should be identified as soon as possible because the early stage does not generally progress to severe disease under adequate treatment (7).

In conclusion, the present study compared definitive and clinical ABPM and found that ABPM is a likely diagnosis when peripheral blood eosinophilia is confirmed and the serum IgE level is increased with concomitant pulmonary infiltration and/or central bronchiectasis. The presence of asthma can indicate ABPM, but it is not essential. A medical interview can reveal a history of sputum expectoration with solid components. The diagnosis should be confirmed by the results of sputum cultures, skin tests, the presence of serum anti-fungal antibody and levels of precipitating antibody against $A f$. A diagnosis of ABPM is established when immunological tests are positive against causative fungi cultivated from airways. However, the causative fungus cannot always be identified, negating the use of specific immunological examinations. Similarly, even when immunological examinations are positive for a specific fungus, it cannot always be cultivated from the patient. Thus, systemic corticosteroid therapy should be initiated and responses carefully followed up in patients with clinically diagnosed ABPM even in the absence of a definitive diagnosis to prevent irreversible pulmonary dysfunction. Early and conventional diagnostic criteria should be established for patients with ABPM.

\section{References}

1. Hinson KF, Moon AJ, Plummer NS. Bronchopulmonary aspergillosis; a review and a report of eight new cases. Thorax 7: 317333, 1952.

2. Akiyama K, Mathison DA, Riker JB, Greenberger PA, Patterson R. Allergic bronchopulmonary candidiasis. Chest 85: 699-701, 1984.

3. Moreno-Ancillo A, Diaz-Pena JM, Ferrer A, et al. Allergic bronchopulmonary cladosporiosis in a child. J Allergy Clin Immunol 97: 714-715, 1996.

4. Muscat I, Oxborrow S, Siddorn J. Allergic bronchopulmonary mycosis. Lancet 1: 1341, 1988.

5. Kamei K, Unno H, Nagao K, Kuriyama T, Nishimura K, Miyaji M. Allergic bronchopulmonary mycosis caused by the basidiomycetous fungus Schizophyllum commune. Clin Infect Dis 18: 305309, 1994.

6. Kawano T, Matsuse H, Iida $\mathrm{K}$, et al. Two cases of allergic bronchopulmonary mycosis caused by Schizophyllum commune in young asthmatic patients. Nihon Kokyuki Gakkai Zasshi (Journal of The Japanese Respiratory Society) 41: 233-236, 2003 (in Japanese, Abstract in English).

7. Kumar R. Mild, moderate, and severe forms of allergic bronchopulmonary aspergillosis. A clinical and serologic evaluation. Chest 124: 890-892, 2003.
8. Rosenberg M, Patterson R, Mintzer R, Cooper BJ, Roberts M, Harris KE. Clinical and immunologic criteria for the diagnosis of allergic bronchopulmonary aspergillosis. Ann Intern Med 86: 405414, 1977.

9. Patterson R, Greenberger PA, Radin RC, Roberts M. Allergic bronchopulmonary aspergillosis: staging as an aid to management. Ann Intern Med 96: 286-291, 1982.

10. Greenberger PA. Allergic bronchopulmonary aspergillosis. J Allergy Clin Immunol 110: 685-692, 2002.

11. Matsuse H, Watanabe $T$, Fujiwara $C$, et al. Six cases of allergic bronchopulmonary aspergillosis. Nihon Kyobu Gakkai Zasshi (Journal of The Japanese Respiratory Society) 32: 836-842, 1994 (in Japanese, Abstract in English).

12. Glancy JJ, Elder JL, McAleer R. Allergic bronchopulmonary fungal disease without clinical asthma. Thorax 36: 345-349, 1981.

13. Henderson AH. Allergic aspergillosis: A review of 32 cases. Tho$\operatorname{rax}$ 23: 501-512, 1968.

14. Tamura A, Hebisawa A, Kurashima A, et al. The use of bronchofiberscopy for diagnosis of allergic bronchopulmonary aspergillosis. Intern Med 36: 865-869, 1997.

15. Greenberger PA. Allergic bronchopulmonary aspergillosis. Allergy II-Principles and Practice-CV Mosby. St. Louis, 1988: 1219-1236.

(C) 2006 The Japanese Society of Internal Medicine

http://www.naika.or.jp/imindex.html 